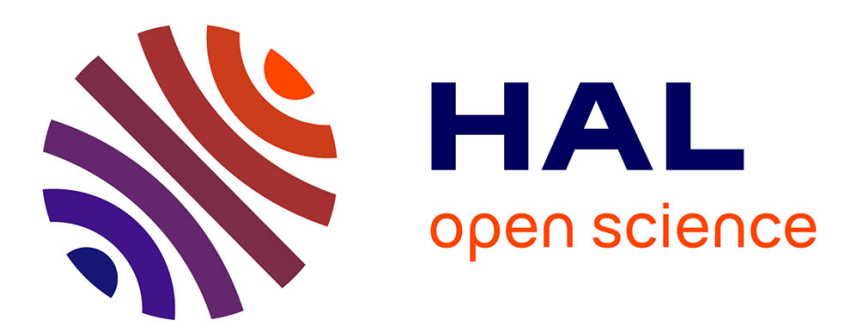

\title{
Chirality-controlled spontaneous currents in spin-orbit coupled superconducting rings
}

\author{
J. Robinson, A. V Samokhvalov, Alexandre I. Buzdin
}

\section{To cite this version:}

J. Robinson, A. V Samokhvalov, Alexandre I. Buzdin. Chirality-controlled spontaneous currents in spin-orbit coupled superconducting rings. Physical Review B: Condensed Matter and Materials Physics (1998-2015), 2019, 99 (18), 10.1103/PhysRevB.99.180501 . hal-02140470

\author{
HAL Id: hal-02140470 \\ https://hal.science/hal-02140470
}

Submitted on 27 May 2019

HAL is a multi-disciplinary open access archive for the deposit and dissemination of scientific research documents, whether they are published or not. The documents may come from teaching and research institutions in France or abroad, or from public or private research centers.
L'archive ouverte pluridisciplinaire HAL, est destinée au dépôt et à la diffusion de documents scientifiques de niveau recherche, publiés ou non, émanant des établissements d'enseignement et de recherche français ou étrangers, des laboratoires publics ou privés. 


\title{
Chirality-controlled spontaneous currents in spin-orbit coupled superconducting rings
}

\author{
J. W. A. Robinson,,$^{1, *}$ A. V. Samokhvalov, ${ }^{2,3, \dagger}$ and A. I. Buzdin ${ }^{1,4,5, \sharp}$ \\ ${ }^{1}$ Department of Materials Science and Metallurgy, University of Cambridge, CB3 OFS Cambridge, United Kingdom \\ ${ }^{2}$ Institute for Physics of Microstructures, Russian Academy of Sciences, 603950 Nizhny Novgorod, GSP-105, Russia \\ ${ }^{3}$ Lobachevsky State University of Nizhni Novgorod, 23 Prospekt Gagarina, 603950 Nizhni Novgorod, Russia \\ ${ }^{4}$ University Bordeaux, LOMA UMR-CNRS 5798, F-33405 Talence Cedex, France \\ ${ }^{5}$ Sechenov First Moscow State Medical University, Moscow 119991, Russia
}

(Received 5 October 2018; revised manuscript received 7 February 2019; published 1 May 2019)

\begin{abstract}
At a superconductor interface with a ferromagnetic insulator (FI), the FI acts to induce a local exchange field within the $\mathrm{S}$ layer, which in the presence of spin-orbit interaction promotes a phase- modulated superconducting state. Here we demonstrate that within a thin superconducting loop that is partially proximitized by a FI, spontaneous currents form with a magnetization-orientation-dependent chirality with sizable shifts in LittleParks oscillations. Furthermore, the critical temperature of the loop is also magnetization-orientation-dependent and conversely, the superconducting transition itself may influence the magnetization direction. More generally, the superconducting region above the FI serves as a "phase battery" and so offers a new device concept for superconducting spintronics.
\end{abstract}

DOI: 10.1103/PhysRevB.99.180501

The interaction of interface superconductivity with materials exhibiting strong spin-orbit coupling [1,2] and magnetic exchange fields [3] offers enormous potential for the discovery and control of new physical phenomena. For example, a homogeneous magnetic exchange field acting at a superconductor/ferromagnet $(\mathrm{S} / \mathrm{F})$ interface induces oscillations in the superconducting order parameter in $S / F / S$ Josephson junctions [4-7] while a nonuniform exchange field in such junctions can lead to electron pair conversion from spin singlet to spin triplet [8-10] and a dependence of the superconducting critical temperature $T_{c}$ on magnetization alignment in $\mathrm{S} / \mathrm{F} / \mathrm{F}$ multilayers [11]. In the absence of inversion symmetry, Rashba spin-orbit (SO) interaction $(\sim[\vec{\sigma} \times \vec{p}] \vec{n}$, where $\vec{n}$ is the unit vector along the axis with broken symmetry [1]) in combination with a magnetic exchange field $\vec{h}$ or Zeeman field offers additional physics with an unusual linear over the gradient of the superconducting order parameter $\Psi$ terms in the Ginzburg-Landau (GL) free energy $\sim[\vec{n} \times \vec{h}](\nabla \Psi) \Psi^{*}$ $[1,12]$. Here we call such coupling the "exchange spin-orbit coupling" or "EXSO" and we see that it may be directly controlled by the exchange field $\vec{h}$ alignment. The EXSO effect induces different types of Larkin-Ovchinnikov-Fulde-Ferrelllike helical phases with a nonzero Cooper pair momentum $\vec{q}$ in the ground state [13-17], which play an important role in Majorana physics [18] and lead to the formation of a $\varphi_{0}$-Josephson junction with a spontaneous phase difference of $\varphi_{0}$ in the ground state [19-21]. Recently, such $\varphi_{0}$-Josephson junctions have been realized experimentally [22].

\footnotetext{
*jjr33@cam.ac.uk

${ }^{\dagger}$ samokh@ipmras.ru

‡alexandre.bouzdine@u-bordeaux.fr
}

We note that in uniform systems the helical phases do not carry a current [23] (this is related with the fact that EXSO modifies the standard expression for the current in GL theory); however, such current-carrying states may appear in nonuniform EXSO systems such as close to a magnetic island on a thin-film superconductor [24] or near an S/F interface within a distance of the London penetration depth of the interface [25].

In this Rapid Communication we demonstrate that the EXSO effect leads to spontaneous currents in a closed superconducting loop in which the superconductor is partially coupled to a ferromagnetic insulator (FI) as shown in Fig. 1. We further demonstrate that the chirality of the current is controllable through the magnetization alignment of the FI. The center of the ring of radius $R$ is at $x=y=0$. In this geometry, we expect a nontrivial interplay between the LittleParks effect and helical current-carrying states. Even in zero external magnetic field, spontaneous currents are generated in the ground state. The study of the Little-Parks oscillations is a powerful technique to probe subtle effects and was recently applied to investigate superconducting symmetry in $\mathrm{Sr}_{2} \mathrm{RuO}_{4}$ microrings [26]. More generally, the revealed mechanism of the spontaneous current generation permits one to realize a phase battery, which can be easily incorporated in different superconducting circuits, enhancing their functionality.

To describe the superconducting loop we apply the very general Ginzburg-Landau (GL) approach which is relevant at a temperature $T$ close to the critical transition $T_{c}$ of the loop. In the presence of the EXSO, the density $f(\mathbf{r})$ of the GL free energy $F=\int f(\mathbf{r}) d^{3} \mathbf{r}$ reads $[14,15]$

$$
\begin{aligned}
f(\mathbf{r})= & a|\psi|^{2}+\gamma|\hat{\mathbf{D}} \psi|^{2}+\frac{b}{2}|\psi|^{4} \\
& +[\overrightarrow{\mathbf{n}} \times \overrightarrow{\mathbf{h}}] \cdot\left[\psi^{*} \varepsilon_{0}(\mathbf{r}) \hat{\mathbf{D}} \psi+\text { c.c. }\right] .
\end{aligned}
$$




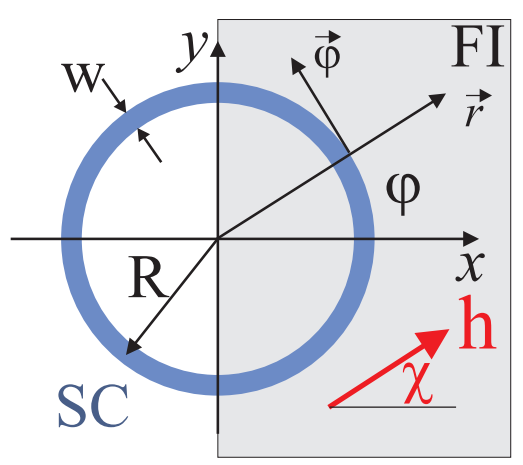

FIG. 1. The geometry of a thin-film superconducting loop proximity coupled to a ferromagnetic insulator (FI) occupying the half plane $x \geqslant 0 . R \gg \xi_{0}$ is the radius of the 1D loop $\left(w \leqslant \xi_{0}\right), \mathbf{h}=h \mathbf{x}_{0}$ is the exchange field, and $(r, \varphi)$ is the polar coordinate system ( $\xi_{0}$, zero-temperature superconducting coherence length).

Here $a=-\alpha\left(T_{c}-T\right), \alpha, b$, and $\gamma>0$ are the standard GL coefficients, $\psi$ is the superconducting order parameter with $\hat{\mathbf{D}}=-i \hbar \boldsymbol{\nabla}+(2 e / c) \mathbf{A}$ the gauge-invariant momentum operator $(e>0), \vec{n}$ is the unit vector in the direction along which the inversion symmetry is broken (pointing in the $z$ direction perpendicular to the $\mathrm{S} / \mathrm{F}$ interface in our case), $\overrightarrow{\mathbf{h}}$ is the exchange field, and $\varepsilon_{0}(\mathbf{r})$ is the EXSO constant. The exchange field $\overrightarrow{\mathbf{h}}$ originates from interactions between the conduction electrons of a metal and local magnetic moments in the FI. The exchange field $\overrightarrow{\mathbf{h}}$ is assumed to be generated in the interfacial S/FI region, where the conduction electron wave functions extend into the ferromagnetic environment of the FI [27-29]. A simple estimate of the characteristic scale of the evanescent tail of a wave function is $a_{0} \sim \hbar / \sqrt{2 m E_{g}} \lesssim$ $2 A$, where $E_{g} \sim 1-2 \mathrm{eV}$ is the typical value of the FI band gap. We consider the case of the thin and narrow superconducting ring with a rectangular cross section (thickness of the superconductor $d$ and the width of the ring $w$ smaller than zero-temperature superconducting coherence length $\xi_{0}$ ), which permit us to use the one-dimensional (1D) GL model. The Rashba SO interaction and Zeeman fields in (1) are localized near the S/F interface and should be effectively averaged over the cross section of the ring. For justification of this averaging procedure see, for example, [4]. Note that we consider the same type of superconducting loop which was successfully used for the observation of the Little-Parks effect (see, for example, [30]). Taking into account that in our case both the SO interaction and exchange field are present at the distance of the order of interatomic distance $a_{0}$ from the $\mathrm{S} / \mathrm{F}$ interface and then they are averaged over the ring thickness $d$, we have $\varepsilon \sim\left(a_{0} / d\right) \varepsilon_{b}$, where $\varepsilon_{b} \sim \gamma v_{\mathrm{so}} / v_{F}^{2}$ is the estimation of the EXSO constant for the bulk superconductor (see, for example, [16]). Here $v_{\text {so }}$ is the characteristic velocity entering in SO Rashba interaction and $v_{F}$ is the Fermi velocity.

For a 1D ring of radius $R$ it is convenient to write the GL free-energy functional (1) using the polar coordinates $(r, \varphi)$, and take into account that the tangential $\varphi$ component $A$ of the vector potential $\mathbf{A}=(0, A, 0)$ is directly related with the magnetic flux $\Phi=2 \pi R A$ enclosed by the superconducting loop. Then the GL free energy then reads

$$
\begin{aligned}
F= & \sigma R \int_{0}^{2 \pi} d \varphi\left\{a|\psi|^{2}+\gamma \frac{\hbar^{2}}{R^{2}}\left|\left(-i \frac{\partial}{\partial \varphi}+\phi\right) \psi\right|^{2}\right. \\
& \left.+\frac{b}{2}|\psi|^{4}+\frac{\hbar}{R} \varepsilon(\varphi)\left[\psi^{*}\left(-i \frac{\partial}{\partial \varphi}+\phi\right) \psi+\text { c.c. }\right]\right\},
\end{aligned}
$$

where $\sigma$ is the cross section of the ring, $\phi=\Phi / \Phi_{0}$ is the enclosed magnetic flux in units of the flux quantum $\Phi_{0}=$ $\pi \hbar c / e$, and

$$
\varepsilon(\varphi)=\varepsilon_{0}[\overrightarrow{\mathbf{n}} \times \overrightarrow{\mathbf{h}}]_{\varphi}=h \varepsilon_{0} \cos (\varphi-\chi)
$$

is a projection on the ring of the Rashba spin-orbit coupling (SOC) for $|\varphi| \leqslant \pi / 2$ and $\varepsilon(\varphi)=0$ otherwise.

To calculate $T_{c}$ we are concerned with the linear equation for the superconducting order parameter, which for the functional (2) can be written as

$$
\left(\frac{\partial}{\partial \varphi}+i \phi\right)^{2} \psi+2 i \lambda(\varphi)\left(\frac{\partial \psi}{\partial \varphi}+i \phi \psi\right)+i \frac{\partial \lambda}{\partial \varphi} \psi=\tau \psi
$$

where $\lambda(\varphi)=\lambda_{\text {so }} \cos (\varphi-\chi), \lambda_{\text {so }}=\varepsilon_{0} h R / \gamma \hbar$ is the EXSO parameter for $|\varphi| \leqslant \pi / 2$, and $\lambda(\varphi)=0$ otherwise. The eigenvalue $\tau$ determines the shift of the transition temperature

$$
T_{c}=T_{c 0}\left(1+\frac{\xi_{0}^{2}}{R^{2}} \tau\right)
$$

with respect to its bulk value $T_{c 0}$. Here $\xi_{0}^{2}=\gamma \hbar^{2} / \alpha T_{c 0}$ is the GL coherence length. Note that averaged exchange field $h_{\mathrm{av}} \sim h a_{0} / d$ and to preserve the superconductivity we need $h_{\mathrm{av}} \lesssim T_{c}$. However, at $v_{\mathrm{so}} / v_{F} \sim 0.1$ we may have for $R / \xi_{0} \sim$ $10-100$ a pretty large spin-orbit parameter $\lambda_{\text {so }} \sim 1-10$.

The current density corresponding to the functional (2) is determined by the relation

$$
j=-\frac{\partial f}{\partial A}=\frac{2 e \gamma \hbar}{R}\left[i \psi \frac{\partial \psi^{*}}{\partial \varphi}-i \psi^{*} \frac{\partial \psi}{\partial \varphi}+2 \lambda \psi^{*} \psi\right]
$$

and we may verify that Eq. (3) guarantees $\operatorname{div} \mathbf{j}=0$.

To explain our main results, we start from a qualitative discussion of the SOC effect on the Little-Parks oscillations. By introducing an effective flux $\tilde{\phi}(\varphi)=\phi+\lambda(\varphi)$, we write Eq. (3) in the following form:

$$
\left(\partial_{\varphi}+i \tilde{\phi}\right)^{2} \psi=\tilde{\tau} \psi,
$$

where $\tilde{\tau}(\varphi)=\tau-\lambda^{2}(\varphi)$. Then the averaged over the ring value

$$
\langle\tilde{\phi}\rangle=\frac{1}{2 \pi} \int_{0}^{2 \pi} d \varphi \tilde{\phi}(\varphi)=\phi+\left(\lambda_{\mathrm{so}} / \pi\right) \cos \chi,
$$

describes the additional shift

$$
\Delta \phi=-\left(\lambda_{\mathrm{so}} / \pi\right) \cos \chi
$$

of the $T_{c}$ maximum of the Little-Parks oscillations.

To provide the full solution of (3) we use the Fourier series expansion for the order parameter

$$
\psi=\sum_{n} \psi_{n} \exp (-i n \varphi)
$$


and EXSO coupling constant

$$
\lambda(\varphi)=\sum_{n} \lambda_{n} \exp (-i n \varphi)
$$

where

$$
\begin{aligned}
\lambda_{n}= & \lambda_{n}^{r}+i \lambda_{n}^{i}=\frac{\lambda_{\mathrm{so}} \cos \chi}{2 \pi} \int_{-\pi / 2}^{\pi / 2} d \varphi \cos \varphi \cos (n \varphi) \\
& +i \frac{\lambda_{\mathrm{so}} \sin \chi}{2 \pi} \int_{-\pi / 2}^{\pi / 2} d \varphi \sin \varphi \sin (n \varphi) .
\end{aligned}
$$

Then we readily obtain from Eq. (3) the systems of coupled algebraic equations for the harmonics $\psi_{n}$

$$
-(n-\phi)^{2} \psi_{n}+\sum_{m} \lambda_{n-m}(n+m-2 \phi) \psi_{m}=\tau \psi_{n} .
$$

Let us consider first the case of the flux smaller than the half quantum $\phi<1 / 2$. In this situation zero harmonics $\psi_{0}$ will dominate $\left(\psi_{n} \ll \psi_{0}\right)$ if $\lambda_{\text {so }}$ is small:

$$
\left(\tau+\phi^{2}+2 \phi \lambda_{0}\right) \psi_{0}=\sum_{n \neq 0} \lambda_{n}^{*}(n-2 \phi) \psi_{n} .
$$

On the other hand for $\psi_{n}$, we have

$$
\left[\tau+(n-\phi)^{2}-2(n-\phi) \lambda_{0}\right] \psi_{n} \approx \lambda_{n}(n-2 \phi) \psi_{0},
$$

which leads to the equation for $\tau$ :

$$
\left(\tau+\phi^{2}+2 \phi \lambda_{0}\right)=\sum_{n \neq 0} \frac{\left|\lambda_{n}\right|^{2}(n-2 \phi)^{2}}{\tau+(n-\phi)^{2}-2(n-\phi) \lambda_{0}} .
$$

Since $\tau \approx-\phi^{2}-2 \lambda_{0} \phi$, then $\tau+(n-\phi)^{2}-2 \lambda_{0}(n-\phi) \approx$ $n\left[n-2\left(\lambda_{0}+\phi\right)\right] \approx n(n-2 \phi)$ and finally we have for the transition temperature

$$
\tau=-\phi^{2}-2 \lambda_{0} \phi+\sum_{n \neq 0}\left|\lambda_{n}\right|^{2}=-\left(\phi+\lambda_{0}\right)^{2}+\lambda_{\mathrm{so}}^{2} / 4 .
$$

In the absence of the external flux $(\phi=0)$ we have the following dependence of the critical temperature $\tau$ on the orientation of the exchange field $\mathbf{h}$ :

$$
\tau=\lambda_{\mathrm{so}}^{2}\left[1-(2 \cos \chi / \pi)^{2}\right] / 4 .
$$

In a general case, if the flux $\phi$ is large $\min |n-\phi|$ occurs for some finite $n_{0}$ and for $\chi=0$ we have similarly

$$
\tau=-\left(\phi+\lambda_{0}-n_{0}\right)^{2}+\lambda_{\mathrm{so}}^{2} / 4 .
$$

The results of the numerical calculations for arbitrary values of $\lambda_{\text {so }}$ are plotted in Fig. 2(a). We see that the strong EXSO interaction shifts the Little-Parks oscillations with a dependence on the orientation of $\mathbf{h}$. One can also observe the stimulation of the superconductivity by spin-orbital interaction: in Fig. 2(b) we compare the dependence of the shifts $\Delta \phi$ of the $T_{c}$ maximum of the Little-Parks oscillations obtained analytically, i.e., using Eq. (6), and numerically. One can see an excellent agreement between the qualitative description (6) and the exact solution of the eigenvalue problem (10). Note that the numerical calculation demonstrates a weak dependence of the maximal $T_{c}$ on the direction of $\mathbf{h}$. In Fig. 3 we show the dependence of the transition temperature $\tau$ on the $\mathbf{h}$ direction $\chi$ in the absence of external flux $(\phi=0)$ for two values of spin-orbital constant $\lambda_{\text {so }}$. The insets in Fig. 3 show spectra of
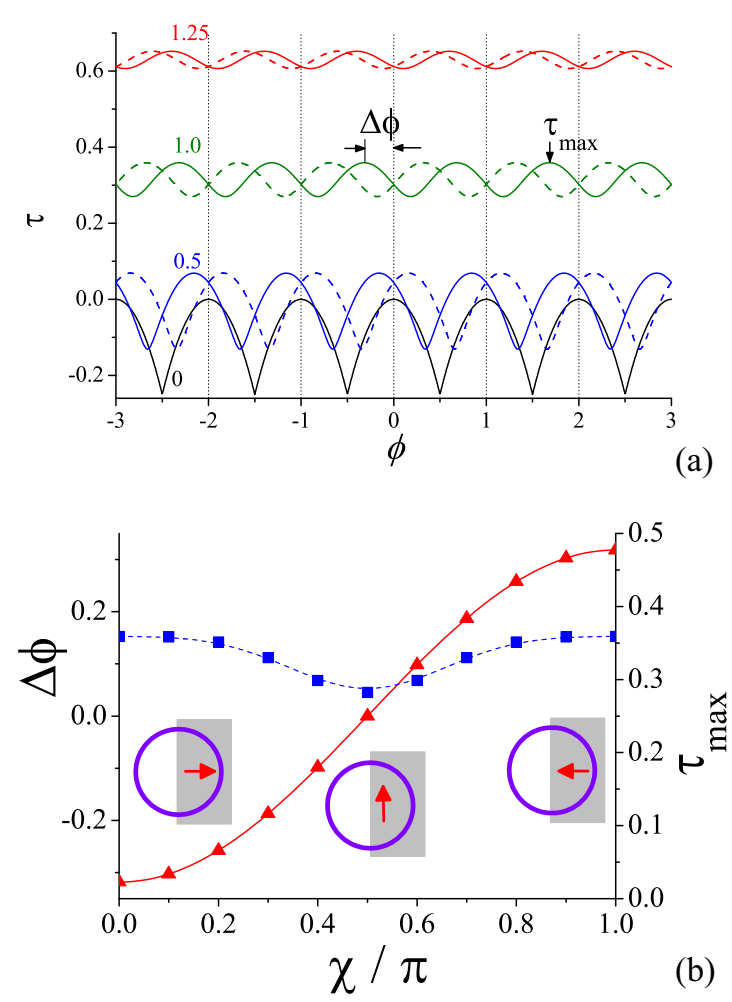

FIG. 2. (a) Little-Parks oscillations for different values of EXSO parameter $\lambda(\varphi)= \pm \lambda_{\text {so }} \cos (\varphi-\chi)$ for $|\varphi| \leqslant \pi / 2$ : $\chi=0$, solid; $\chi=\pi$, dashed. The numbers near the curves denote the corresponding values of the spin-orbit constant $\lambda_{\text {so }}$. (b) Dependence of the shifts of the Little-Parks oscillations $\Delta \phi(\Delta)$ and the maximal transition temperature $\tau_{\max }(--)$ [see panel (a) for comparison] on the angle $\chi$ for $\lambda_{\text {so }}=1$. Solid red line shows the dependence described by Eq. (6).

orbital harmonics for the selected values of $\lambda_{\text {so }}$ at $\chi=0$. For small values of the EXSO constant the zero harmonic prevails (left inset in Fig. 3), and $\tau(\chi)$ dependence is well described by expression (11). The spin-orbital interaction results in generation of harmonics with nonzero orbital momenta. As a result

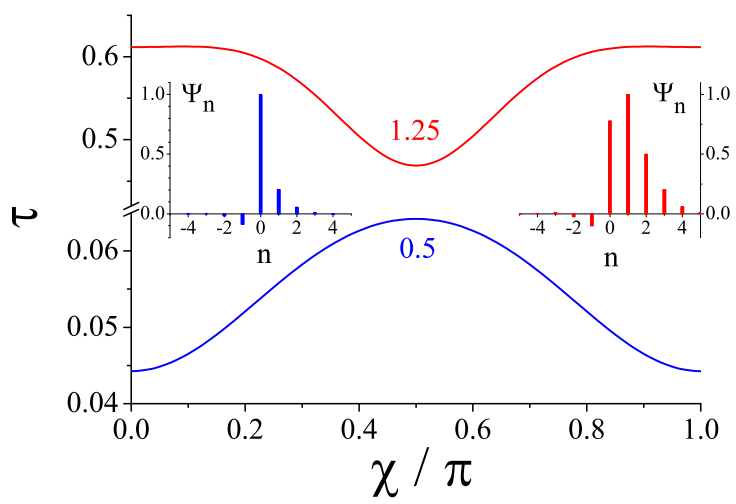

FIG. 3. Dependence of the transition temperature $\tau$ on the angle $\chi$ for $\lambda_{\text {so }}=0.5$ (blue solid line) and $\lambda_{\text {so }}=1.25$ (red solid line) obtained by the numerical solution of the eigenvalue problem (10) for $\phi=0$. The insets show the spectra of orbital harmonics for $\chi=0$ and different values of the spin-orbit constant: left, $\lambda_{\text {so }}=0.5$; right, $\lambda_{\text {so }}=1.25$. 


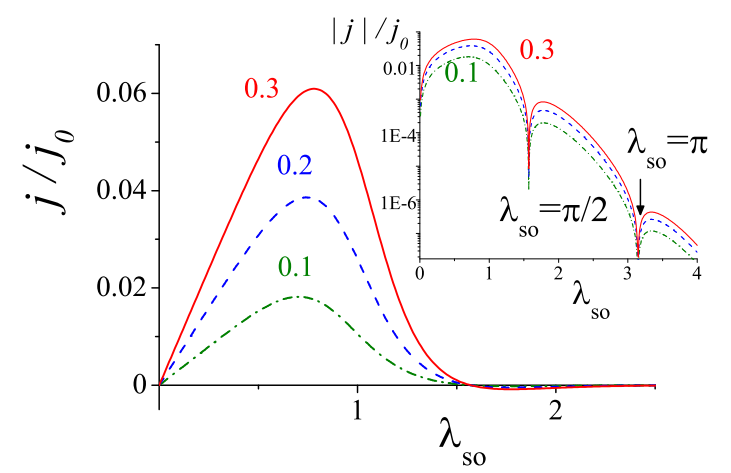

FIG. 4. The dependence of the current density $j$ on spinorbital constant $\lambda_{\text {SO }}$ for different temperatures $T<T_{c} \quad\left[j_{0}=\right.$ $\left.4 e(\hbar / R)^{3} \gamma^{2} / b\right]$. The numbers by the curves correspond to the dimensionless shift $\Delta \tau=\left(R / \xi_{0}\right)^{2}\left(T_{c}-T\right) / T_{c}$ in $T$ with respect to the transition (4).

the spectrum of orbital harmonics spreads with an increase of the spin-orbital interaction, and a dominant harmonic has a nonzero orbital momenta (right inset in Fig. 3).

We now calculate the current for the case of spin-orbit interaction in the absence of the applied field with $\phi=0$ and the exchange field oriented along the $x$ axis $(\chi=0)$. We calculate the current at $\varphi=\pi$, where the expression for the current density has the standard form (5) with $\lambda=0$ and

$$
j=-4 e \frac{\gamma \hbar}{R} \sum_{n m} n(-1)^{n-m} \operatorname{Re}\left[\psi_{n} \psi_{m}^{*}\right] \approx 4 e \frac{\gamma \hbar \lambda_{\text {so }}}{\pi R} \psi_{0}^{2} .
$$

Naturally, the current vanishes for the exchange field oriented along the $y$ axis $(\chi=\pi / 2)$. Figure 4 shows the dependence of the current density $j$ (12) on the spin-orbital constant $\lambda_{\text {SO }}$ for different temperatures $T<T_{c}$. One can see that even in the absence of an external magnetic field the superconducting transition occurs in the current-carrying state with nonzero orbital momenta. The sign and magnitude of the spontaneous supercurrent due to spin-orbital interaction is determined by the EXSO constant with a maximum at $\lambda_{\text {so }} \sim 1$.

We have considered above the case of a thin superconducting ring; however, the effect of the phase accumulation due to EXSO coupling (described by the constant $\varepsilon \sim h \varepsilon_{0}$ ) is very general and can play the role of a superconducting phase battery. Indeed, let us consider the superconducting loop at temperatures below $T_{c}$ where modulus $|\psi|$ of the order parameter is constant. In the case of the infinite wire in the presence of the EXSO coupling the phase modulated solution is realized $\psi=|\psi| \exp (i \varphi)$ with $\varphi=q_{0} l$ and $q_{0}=$ $h \varepsilon_{0} / \gamma \hbar$ (l, coordinate along the wire). This solution, however, corresponds to the absence of superconducting current

$$
j=4 e \hbar \gamma|\psi|^{2}\left(\frac{\partial \varphi}{\partial l}-q_{0}\right)=0 .
$$

The boundary condition at the interface between modulated $(\varepsilon \neq 0)$ and usual $(\varepsilon=0)$ superconducting phase corresponds simply to the continuity of the current. Let us consider a generic example of the superconducting wire loop consisting of a region of length $L_{h}$ with an exchange field and $L_{0}$ without an exchange field [see Fig. 5(a)]. The solution in the first region is $|\psi| \exp \left(i q_{1} l\right)$ and the corresponding cur-
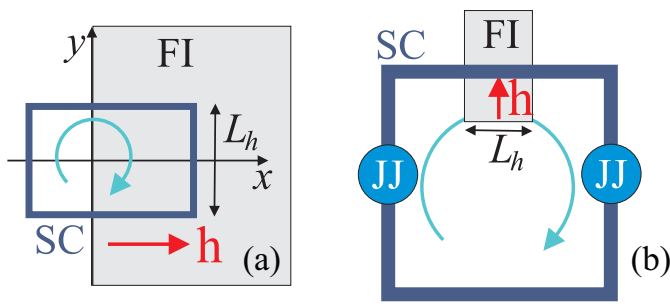

FIG. 5. (a) Schematic representation of a thin superconducting loop with the EXSO interaction. (b) dc SQUID with the EXSO "phase battery."

rent is $j_{1}=4 e \hbar \gamma|\psi|^{2}\left(q_{1}-q_{0}\right)$. The solution in the second region is $|\psi| \exp \left(i q_{2} l\right)$ and the corresponding current is $j_{2}=$ $4 e \hbar \gamma|\psi|^{2} q_{2}$. From the continuity of the current we have $q_{1}-q_{0}=q_{2}$. The total phase accumulation over the loop will be $\Delta \varphi=q_{1} L_{h}+q_{2} L_{0}$ and should be equal to $2 \pi n$. From this condition we find $q_{2}=\left(2 \pi n-q_{0} L_{h}\right) /\left(L_{h}+L_{0}\right)$, which means the spontaneous generation of the current (except the special cases when the phase accumulation in the active $L_{h}$ region is an integer of $2 \pi$, i.e., $q_{0} L_{h}=2 \pi n$ ). The actual choice of $n$ is dictated by the minimization of the current $j_{1}=j_{2}$ and so the associated magnetic energy, i.e., $\min \left|2 \pi n-q_{0} L_{h}\right|$. Therefore we may conclude that the part of the wire with EXSO coupling serves as a "phase battery."

In conclusion, we have demonstrated that the spin-orbit interaction in combination with a magnetic exchange field (EXSO) may induce nondissipative currents in superconducting loops with a chirality that is controllable through the magnetization alignment. Superconducting elements with EXSO may generate spontaneous phase shifts governed by magnetic moment orientation and by introducing the EXSO wire $L_{h}$ in a dc SQUID [see Fig. 5(b)] we can mimic the role of external flux $2 \pi \Phi / \Phi_{0}=q_{0} L_{h}$ which opens the way to creating "quiet" qubits [31], similar to that which has been recently fabricated on the basis of the $\pi$ junction [32]. Suitable FI materials for the proposed experiments here include EuS [33] and GdN [34] and potentially oxides such as praseodymium calcium manganese oxide [35]. In [34] the effective averaging of the exchange field from the high-quality S/FI interface was experimentally demonstrated. The interface with the ferromagnetic metal may also be appropriate for the EXSO induction, provided its thickness is much smaller that the $S$ thickness (to avoid strong pair-breaking effect). We believe that the most suitable geometry for the experimental verification of our prediction is a loop/square with size of the order of $10 \xi_{0}$ and width and thickness less than $\xi_{0}$. Finally, we note that the EXSO interaction between magnetic and superconducting subsystems may, in principle, generate an inverse effect in which the superconducting transition provokes reorientation of the magnetic moment.

J.W.A.R. acknowledges funding from the EPSRC through an International Network Grant and Programme Grant (Grants No. EP/P026311/1 and No. EP/N017242/1). J.W.A.R. also acknowledges funding from the Royal Society through a University Research Fellowship and A.I.B. acknowledges funding from the Leverhulme Trust and EU Network COST CA16218 (NANOCOHYBRI). This work was supported by Russian 
Foundation for Basic Research (RFBR) under Grants No. 17-52-12044-NNIO and No. 18-02-00390 (A.V.S.) and the
French ANR project SUPERTRONICS and OPTOFLUXONICS (A.I.B.).
[1] V. P. Mineev and M. Sigrist, in Non-Centrosymmetric Superconductors, edited by E. Bauer and M. Sigrist, Lecture Notes in Physics, Vol. 847 (Springer, Berlin, Heidelberg, 2012), pp. 129-154.

[2] M. Smidman, M. B. Salamon, H. Q. Yuan, and D. F. Agterberg, Rep. Prog. Phys. 80, 036501 (2017).

[3] J. Linder and J. W. A. Robinson, Nat. Phys. 11, 307 (2015); M. G. Blamire and J. W. A. Robinson, J. Phys.: Condens. Matter 26, 453201 (2014); M. Eschrig, Phys. Today 64(1), 43 (2011).

[4] A. I. Buzdin, Rev. Mod. Phys. 77, 935 (2005); F. S. Bergeret, A. F. Volkov, and K. B. Efetov, ibid. 77, 1321 (2005).

[5] T. Kontos, M. Aprili, J. Lesueur, and X. Grison, Phys. Rev. Lett. 86, 304 (2001).

[6] V. V. Ryazanov, V. A. Oboznov, A. Yu. Rusanov, A. V. Veretennikov, A. A. Golubov, and J. Aarts, Phys. Rev. Lett. 86, 2427 (2001); V. A. Oboznov, V. V. Bol'ginov, A. K. Feofanov, V. V. Ryazanov, and A. I. Buzdin, ibid. 96, 197003 (2006).

[7] J. W. A. Robinson, S. Piano, G. Burnell, C. Bell, and M. G. Blamire, Phys. Rev. Lett. 97, 177003 (2006); J. W. A. Robinson, Z. H. Barber, and M. G. Blamire, Appl. Phys. Lett. 95, 192509 (2009); J. W. A. Robinson, S. Piano, G. Burnell, C. Bell, and M. G. Blamire, IEEE Trans. Appl. Supercond. 17, 641 (2007); Phys. Rev. B 76, 094522 (2007); S. Piano, J. W. A. Robinson, G. Burnell, and M. G. Blamire, Eur. Phys. J. B 58, 123 (2007).

[8] J. W. A. Robinson, J. D. S. Witt, and M. G. Blamire, Science 329, 59 (2010); I. T. M. Usman, K. A. Yates, J. D. Moore et al., Phys. Rev. B 83, 144518 (2011); F. Chiodi, J. D. S. Witt, R. G. J. Smits et al., Europhys. Lett. 101, 37002 (2013); A. Di Bernardo, Z. Salman, X. L. Wang et al., Phys. Rev. X 5, 041021 (2015); N. Banerjee, C. B. Smiet, R. G. J. Smits et al., Nat. Commun. 5, 3048 (2014); G. B. Halasz, M. G. Blamire, and J. W. A. Robinson, Phys. Rev. B 84, 024517 (2011); J. D. S. Witt, J. W. A. Robinson, and M. G. Blamire, ibid. 85, 184526 (2012); J. W. A. Robinson, F. Chiodi, M. Egilmez et al., Sci. Rep. 2, 699 (2012); N. Banerjee, J. W. A. Robinson, and M. G. Blamire, Nat. Commun. 5, 4771 (2014).

[9] T. S. Khaire, M. A. Khasawneh, W. P. Pratt, and N. O. Birge, Phys. Rev. Lett. 104, 137002 (2010); C. Klose, T. S. Khaire, Y. Wang et al., ibid. 108, 127002 (2012).

[10] M. S. Anwar, M. Veldhorst, A. Brinkman, and J. Aarts, Appl. Phys. Lett. 100, 052602 (2012).

[11] P. V. Leksin, N. N. Garif'yanov, I. A. Garifullin, Ya. V. Fominov, J. Schumann, Y. Krupskaya, V. Kataev, O. G. Schmidt, and B. Buchner, Phys. Rev. Lett 109, 057005 (2012); X. L. Wang, A. Di Bernardo, N. Banerjee, A. Wells, F. S. Bergeret, M. G. Blamire, and J. W. A. Robinson, Phys. Rev. B 89, 140508(R) (2014); A. Singh, S. Voltan, K. Lahabi, and J. Aarts, Phys. Rev. X 5, 021019 (2015); Y. Gu, G. B. Halasz, J. W. A. Robinson, and M. G. Blamire, Phys. Rev. Lett. 115, 067201 (2015); Z. Feng, J. W. A. Robinson, and M. G. Blamire, Appl. Phys. Lett. 111, 042602 (2017); A. Srivastava, L. A. B. Olde Olthof, A. Di Bernardo et al., Phys. Rev. Appl. 8, 044008 (2017).
[12] V. M. Edelstein, J. Phys.: Condens. Matter 8, 339 (1996).

[13] V. Barzykin and L. P. Gor'kov, Phys. Rev. Lett. 89, 227002 (2002).

[14] K. V. Samokhin, Phys. Rev. B 70, 104521 (2004).

[15] R. P. Kaur, D. F. Agterberg, and M. Sigrist, Phys. Rev. Lett. 94, 137002 (2005).

[16] O. Dimitrova and M. V. Feigel'man, Phys. Rev. B 76, 014522 (2007).

[17] V. M. Edelstein, Phys. Rev. Lett. 75, 2004 (1995).

[18] J. Alicea, Rep. Prog. Phys. 75, 076501 (2012).

[19] A. Buzdin, Phys. Rev. Lett. 101, 107005 (2008); F. Konschelle and A. Buzdin, ibid. 102, 017001 (2009).

[20] I. V. Krive, L. Y. Gorelik, R. I. Shekhter, and M. Jonson, Low Temp. Phys. 30, 398 (2004).

[21] A. A. Reynoso, G. Usaj, C. A. Balseiro, D. Feinberg, and M. Avignon, Phys. Rev. Lett. 101, 107001 (2008).

[22] D. B. Szombati, S. Nadj-Perge, D. Car, S. R. Plissard, E. P. A. M. Bakkers, and L. P. Kouwenhoven, Nat. Phys. 12, 568 (2016).

[23] D. F. Agterberg, in Non-Centrosymmetric Superconductors, edited by E. Bauer and M. Sigrist, Lecture Notes in Physics, Vol. 847 (Springer, Berlin, Heidelberg, 2012) pp. 155-170.

[24] S. S. Pershoguba, K. Björnson, A. M. Black-Schaffer, and A. V. Balatsky, Phys. Rev. Lett. 115, 116602 (2015).

[25] S. Mironov and A. Buzdin, Phys. Rev. Lett. 118, 077001 (2017).

[26] Yuuki Yasui, Kaveh Lahabi, Muhammad Shahbaz Anwar, Yuji Nakamura, Shingo Yonezawa, Takahito Terashima, Jan Aarts, and Yoshiteru Maeno, Phys. Rev. B 96, 180507(R) (2017); J. Jang, D. G. Ferguson, V. Vakaryuk, R. Budakian, S. B. Chung, P. M. Goldbart, and Y. Maeno, Science 331, 186 (2011).

[27] T. Tokuyasu, J. A. Sauls, and D. Rainer, Phys. Rev. B 38, 8823 (1988).

[28] Yu. A. Izyumov, Yu. N. Proshin, and M. G. Khusainov, Phys. Usp. 45, 109 (2002).

[29] E. Strambini, V. N. Golovach, De Simoni, J. S. Moodera, F. S. Bergeret, and F. Giazotto, Phys. Rev. Mater. 1, 054402 (2017).

[30] D. Gurovich, K. S. Tikhonov, D. Mahalu, and D. Shahar, Phys. Rev. B 91, 174505 (2015); O. J. Sharon, A. Sharoni, J. Berger, A. Shaulov, and Y. Yeshurun, J. Phys.: Conf. Ser. 969, 012047 (2018).

[31] L. B. Ioffe, V. B. Geshkenbein, M. V. Feigel'man, A. L. Fauchère, G. Blatter, Nature (London) 398, 679 (1999).

[32] K. Feofanov, V. A. Oboznov, V. V. Bol'ginov, J. Lisenfeld, S. Poletto, V. V. Ryazanov, A. N. Rossolenko, M. Khabipov, D. Balashov, A. B. Zorin, P. N. Dmitriev, V. P. Koshelets, and A. V. Ustinov, Nat. Phys. 6, 593 (2010).

[33] X. Hao, J. S. Moodera, and R. Meservey, Phys. Rev. Lett. 67, 1342 (1991).

[34] A. Pal and M. G. Blamire, Phys. Rev. B 92, 180510(R) (2015); A. Pal, Z. H. Barber, J. W. A. Robinson, M. G. Blamire, Nat. Commun. 5, 3340 (2014); Y. Zhu, A. Pal, M. G. Blamire, and Z. H. Barber, Nat Mater. 16, 195 (2017).

[35] S. Komori, A. Di Bernardo, A. I. Buzdin, M. G. Blamire, and J. W. A. Robinson, Phys. Rev. Lett. 121, 077003 (2018). 